\title{
Use of X-band marine radars as a remote sensing system to survey wind-generated waves
}

\author{
J. C. Nieto Borge \\ Universidad de Alcalá, Spain
}

\begin{abstract}
This work deals with the use of conventional X-band marine radars to characterize sea state information. Thus, those radar systems are able to scan the sea surface in the spatio-temporal domain by acquiring temporal sequences of radar images of the ocean. The paper describes the different algorithms to obtain the estimation of the wave spectra from the radar data sets and the related sea state parameters. In addition, new recently developed techniques are described in the text that permit us to estimate the wave elevation maps of the sea surface $\eta(x, y, t)$, as well as individual wave properties, such as the wave groupiness evolution in space and time.
\end{abstract}

Keywords: marine radar, wind-generated waves, remote sensing.

\section{Introduction}

Wave information is usually characterised from a time series of the sea surface elevation measured at a specific ocean position. These measurements are carried out by in-situ sensors such as anchored buoys. The use of point measurements assumes that the obtained wave information is representative of not only the mooring point, but also of a particular area of the ocean. That assumption is not generally correct, particularly in coastal waters, where coastal effects like wave refraction, diffraction, shoaling etc. take place. Under these conditions, the sea state can vary significantly in the area of interest. Additional to those punctual measurements described above, the imaging of the sea surface based on microwave remote sensing techniques provides information about the sea state variability in the area of interest. One of these techniques is based on the use of ordinary Xband marine radars to analyze the spatial and temporal behavior of ocean wave fields [1-5]. The measurement of ocean waves with marine radars is based on the 


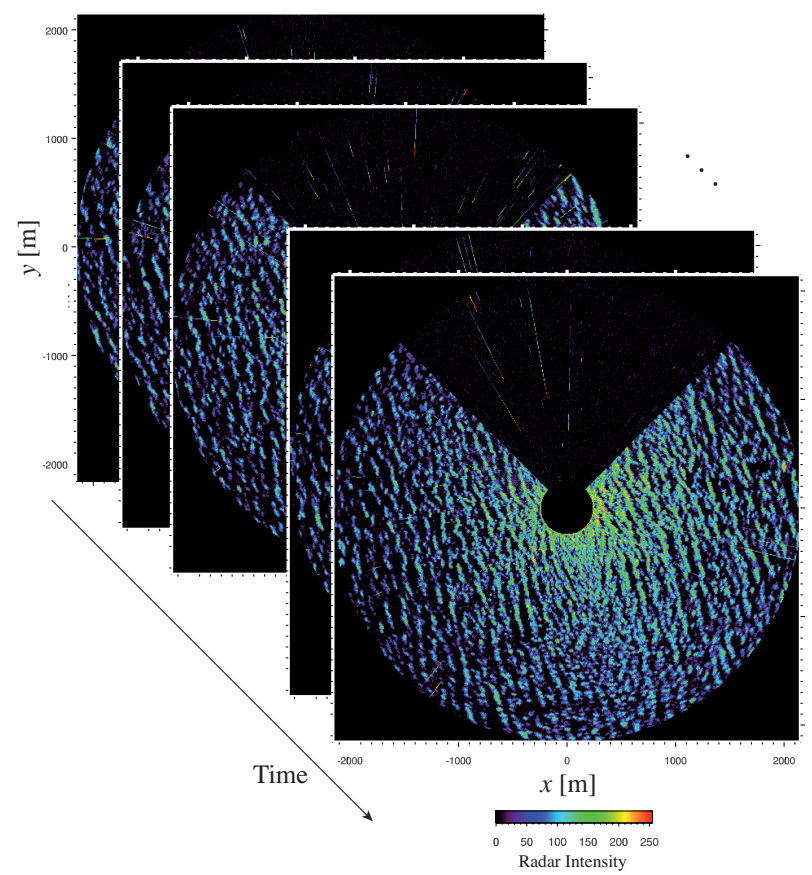

Figure 1: Example of temporal sequence of sea clutter images.

spatial and temporal structure analysis of the sea surface radar images. These radar images are caused by the interaction of the electromagnetic waves transmitted by the radar antenna with the sea surface ripples caused by the local wind [6-9]. This interaction produces a backscatter of the electromagnetic fields and, therefore, an image pattern in the radar display unit, which is commonly known by sailors as sea clutter. Fig. 1 shows an example of a sea clutter image taken by a marine radar on board a moving vessel in the North Sea.

\section{Spectral representation of sea states}

Sea states are regarded as wind-generated wave fields with invariant statistical properties at a given sea surface position $\mathbf{r}=(x, y)$ and at time $t$. Those wave fields are homogeneous in the spatial dependence and stationary in their temporal evolution. Under these assumptions, the sea surface elevation $\eta(\mathbf{r}, t)$ has the following spectral representation

$$
\eta(\mathbf{r}, t)=\int_{\Omega_{\mathbf{k}, \omega}} e^{i(\mathbf{k} \cdot \mathbf{r}-\omega t)} d Z(\mathbf{k}, \omega),
$$

where $\mathbf{k}=\left(k_{x}, k_{y}\right)$ is the two-dimensional wave number, and $\omega$ is the angular frequency. The integration domain $\Omega_{\mathbf{k}, \omega}=\left[-k_{x_{c}}, k_{x_{c}}\right] \times\left[-k_{y_{c}}, k_{y_{c}}\right] \times\left[-\omega_{c}, \omega_{c}\right]$ 
is defined from the range of wavelengths $\lambda=2 \pi / k$ and periods $T=2 \pi / \omega$ of the wind-generated waves. The amplitudes $d Z(\mathbf{k}, \omega)$ are the so-called random spectral measures, which lead the three-dimensional wave spectrum as $F^{(3)}(\mathbf{k}, \omega) d^{2} k d \omega=$ $\mathbb{E}\left[d Z(\mathbf{k}, \omega) d Z^{*}(\mathbf{k}, \omega)\right]$, where $\mathbb{E}$ is the expectation operator. Ocean waves are dispersive, and under the assumptions of the linear wave theory, the dispersion relation is given by

$$
\omega=\varpi(\mathbf{k})=\sqrt{g k \tanh (k d)}+\mathbf{k} \cdot \mathbf{U}=\varpi_{0}(k)+\mathbf{k} \cdot \mathbf{U},
$$

being $g$ the acceleration of gravity, $d$ is the water depth, and $\mathbf{U}=\left(U_{x}, U_{y}\right)$ is the current of encounter responsible of a Doppler shift term in frequency in Eq. (2) given by the dot product $\mathbf{k} \cdot \mathbf{U}$.

\subsection{Additional spectral representation of sea states}

Integrating $F^{(3)}(\mathbf{k}, \omega)$ over all the positive wave frequencies, the unambiguous two-dimensional wave number spectrum $F^{(2)}(\mathbf{k})$ is derived

$$
F^{(2)}(\mathbf{k})=2 \int_{0}^{\omega_{c}} F^{(3)}(\mathbf{k}, \omega) d \omega .
$$

Taking into account the dispersion relation (2), and the two-dimensional wave number spectrum $F^{(2)}(\mathbf{k})$ given by Eq. (3), other spectral densities can be derived. One of the most common spectral representations used is the directional spectrum $E(\omega, \theta)$, which is given by

$$
E(\omega, \theta)=F^{(2)}(\mathbf{k}(\omega, \theta)) k(\omega, \theta) \frac{\partial k(\omega, \theta)}{\partial \omega},
$$

where $\theta=\tan ^{-1}\left(k_{y} / k_{x}\right)$ is the wave propagation direction. The term $k(\omega, \theta)$. $\partial k(\omega, \theta) / \partial \omega$ in Eq. (4) is the Jacobian needed to change the coordinates from the $\left(k_{x}, k_{y}\right)$-space into the $(\omega, \theta)$-space [1].

\section{Estimation of the wave spectrum from radar images}

As mentioned above, the procedure to analyze wave fields using a marine radar is based on the acquisition of temporal sequences composed of $N_{t}$ consecutive images of the sea clutter. The sampling time $\Delta t$ of this temporal sequence of images is given by the antenna rotation period. The spatial resolutions ( $\Delta x$ and $\Delta y$ ) of each image depends on the azimuthal and the range resolution of the radar system.

When analyzing sea clutter data sets it can be realized that all these phenomena contribute with additional spectral components to the spectrum of the sea clutter time series. These additional components do not belong to the wave field imaged by the radar [6-8]. Hence, the spectrum of the sea clutter time series, commonly known in the scientific literature as image spectrum, has to be processed in 


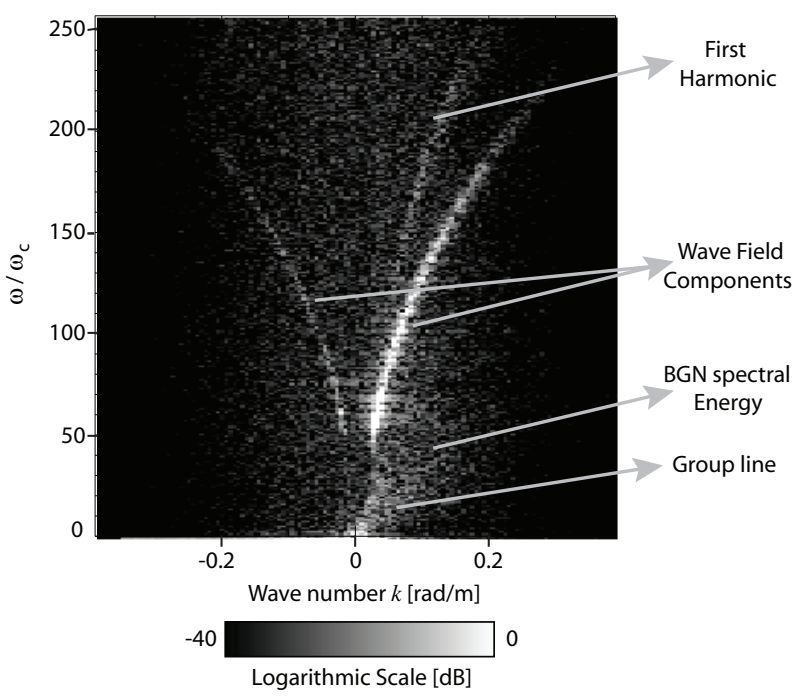

Figure 2: Example of a two-dimensional transect of an image spectrum $I^{(3)}(\mathbf{k}, \omega)$ (see text for explanation). The transect is defined within the $\left(\mathbf{k}_{p}, \omega\right)$ domain, where $\mathbf{k}_{p}$ is the wave number vector along the spectral-peak wave propagation direction. The value of the cut-off angular frequency is $\omega_{c}=1.78 \mathrm{rad} \mathrm{s}^{-1}$.

order to derive reliable estimation of the wave spectrum and the related sea state parameters. As mentioned above, once the temporal sequence of sea clutter images $\zeta(\mathbf{r}, t)$ is acquired, the first step of the analysis is to apply a three-dimensional Fourier decomposition to estimate the so-called image spectrum $I^{(3)}(\mathbf{k}, \omega)$. As mentioned above, the different X-band radar imaging mechanisms contribute with additional spectral $(\mathbf{k}, \omega)$-components different from the components only due to the imaged wave field. These contributions to the image spectrum $I^{(3)}(\mathbf{k}, \omega)$ can be summarized as:

- Static and quasi-static spectral components due to long range radar imaging dependences given by the radar equation [1].

- Wave field components [1].

- Higher harmonics of the wave components due to nonlinear mechanisms in the marine radar imagery [9-11].

- Background noise spectral energy (BGN) due to the roughness of the sea surface $[6,9]$.

- Additional contributions in lower frequency planes, such as the group line [12].

Fig. 2 illustrates an example of image spectrum showing those spectral components mentioned above. This measurement was taken using a land-based radar station on top of a cliff on the Northern coast of Spain (Bay of Biscay). This is a swell dominated area where the long waves reach the cliff walls and some part 
of this incoming wave energy is reflected. Hence, the radar is measuring in that case a bimodal sea state. This bimodality can be appreciated in the two opposite branches of the dispersion relation, where the weaker branch corresponds to the reflected wave components. Therefore, in that case, the quantification of the ratio of reflected energy to the incoming energy can be easily obtained from these kinds of sensors.

To derive the estimation of the wave spectrum, it is necessary to apply an inversion modeling technique. The basic assumption of this inversion modeling algorithms is the existence of the dispersion relation $\varpi(\mathbf{k})$ given by Eq. (2).

\subsection{Inversion modeling technique to derive the wave spectrum}

Once the image spectrum $I^{(3)}(\mathbf{k}, \omega)$ is obtained, the next step is to apply the inversion model to derive the wave spectrum and the related sea state parameters. This modeling technique is composed of the following steps:

1. Applying low-pass filter to $I^{(3)}(\mathbf{k}, \omega)$ to remove the static and quasi-static patterns [1].

2. Estimation of the current of encounter: the estimation of $\mathbf{U}$ is carrying out analyzing the distribution of the $(\mathbf{k}, \omega)$ spectral components. The physical model applied is the linear wave theory, and the dispersion relation (2). Thus, the current of encounter $\mathbf{U}=\left(U_{x}, U_{y}\right)$ can be estimated by minimizing the following functional $[1,13]$ :

$$
\mathcal{V}=\sum_{j=1}^{N_{r}}\left[\omega_{j}-\varpi_{0}\left(k_{j}\right)-k_{x_{j}} U_{x}-k_{y_{j}} U_{y}\right]^{2},
$$

where $k_{j}=\sqrt{k_{x_{j}}^{2}+k_{y_{j}}^{2}}$, and $\varpi_{0}(k)$ is the dispersion relation (2) without the presence of current of encounter U. $N_{r}$ is the number $(\mathbf{k}, \omega)$ points whose spectral energy is due to the wave field and not due to other effects in the radar imaging. The computation of $\mathbf{U}$ from the functional (5) can be improved using an additional iterative scheme [14].

3. Filtering the spectral energy outside the dispersion shell $[1,6,10,13]$ :

$$
F_{f}^{(3)}(\mathbf{k}, \omega)=\int_{\Omega_{\mathbf{k}, \omega}} I^{(3)}\left(\mathbf{k}^{\prime}, \omega^{\prime}\right) \delta\left(\mathbf{k}^{\prime}-\mathbf{k}\right) \cdot \delta\left(\omega^{\prime}-\omega\left(\mathbf{k}^{\prime}\right)\right) d^{2} k^{\prime} d \omega^{\prime}
$$

where $\delta(\cdot)$ is the Dirac's delta.

4. Application of the modulation transfer function: for horizontal polarization and at grazing incidence, a difference between the image spectra from marine radar imagery and the corresponding spectra from in situ sensors can be observed. This difference is due to radar wave imaging mechanisms like, for example, shadowing and/or tilt modulation $[10,11]$ that are not considered in the band-pass filter (6). This effect can be minimized by using a so-called modulation transfer function $[6,15,16]$. The modulation transfer function $\mathcal{T}(\mathbf{k})$ for marine radar has been derived empirically [9] as 


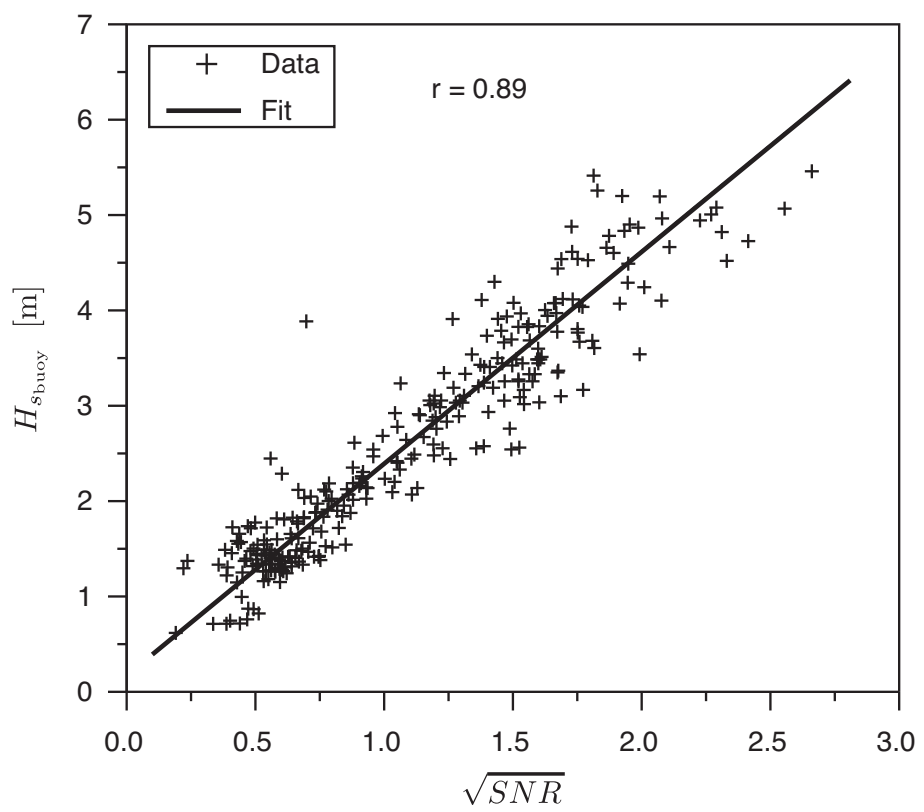

Figure 3: Scatter plot of the $\sqrt{S N R}$ and the buoy $H_{s}$ estimation using wave energy and the $B G N$ energy outside the dispersion shell. The measurements were carried out in the Northern North Sea.

$\mathcal{T}(\mathbf{k})=k^{-1.2}$. Thus, the three-dimensional wave spectrum $\tilde{F}^{(3)}(\mathbf{k}, \omega)$ is given by

$$
\tilde{F}^{(3)}(\mathbf{k}, \omega)=\mathcal{T}(\mathbf{k}) \cdot F_{f}^{(3)}(\mathbf{k}, \omega)
$$

5. Significant wave height estimation: marine radars provide images coded in relative values of grey scales rather than values of physical parameters related to the backscattering phenomenon, such as the radar cross section. Hence, the spectral estimation of the wave field (7) is not properly scaled to derive the significant wave height $H_{s}$. Analyzing the structure of the image spectrum $I^{(3)}(\mathbf{k}, \omega)$ it can be seen that $H_{s}$ can be estimated in a similar way to Synthetic Aperture Radar (SAR) systems [17]. For this particular case, the method has to be extended to the three dimensions of the image spectrum. Under these conditions, the significant wave height has a linear dependence with the root squared of the Signal-to-Noise ratio $S N R[6,9,18]$,

$$
H_{s}=c_{0}+c_{1} \sqrt{S N R}
$$




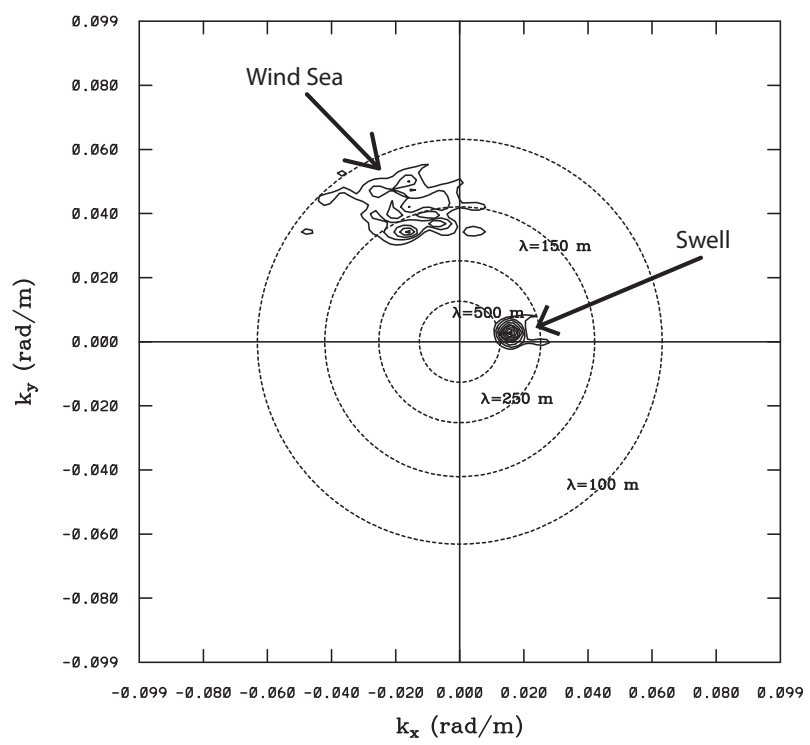

Figure 4: Bimodal wave number spectrum $F^{(2)}(\mathbf{k})$ measured by a marine radar in the North Sea. The measurement was taken in the vicinity of the Norwegian island of Utsira.
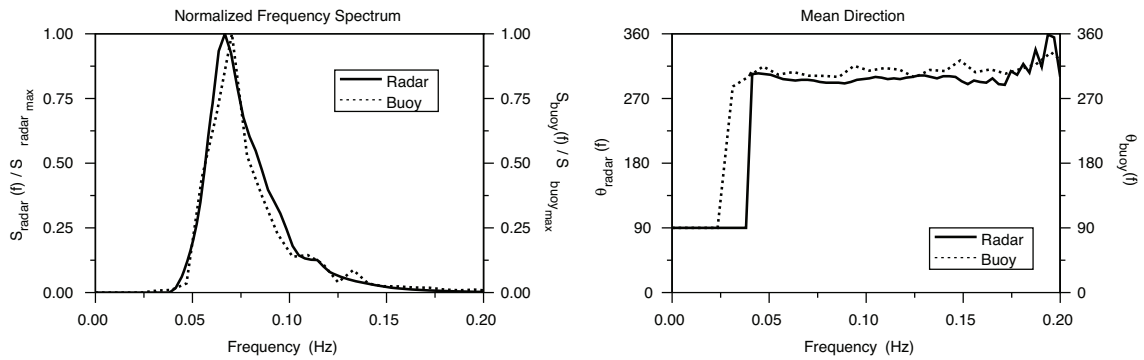

Figure 5: Comparison of the frequency spectrum $S(f)$ (left) and the mean wave direction $\bar{\theta}(f)$ (right) derived from a marine radar (solid line) and buoy data (dotted line).

where $c_{0}$ and $c_{1}$ are calibration constants $[9,18]$. The factor $S N R$ is defined as [9]

$$
S N R=\frac{\int_{\Omega_{\mathbf{k}, \omega}} \tilde{F}^{(3)}(\mathbf{k}, \omega) d^{2} k d \omega}{\int_{\Omega_{\mathbf{k}, \omega}} F_{B N G}^{(3)}(\mathbf{k}, \omega) d^{2} k d \omega},
$$




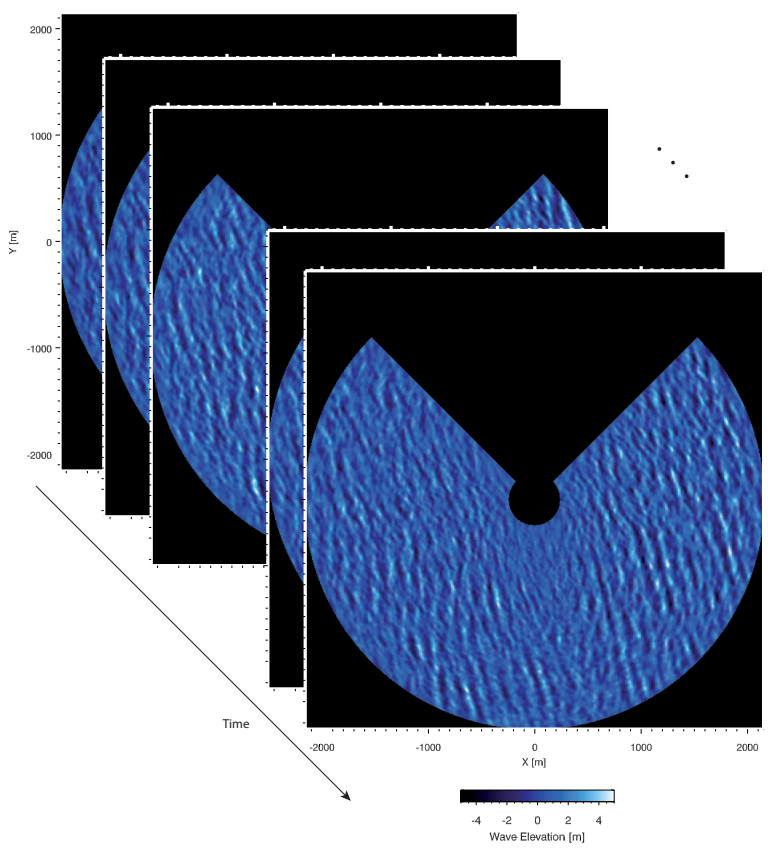

Figure 6: Estimation of the sea surface wave elevation $\eta(\mathbf{r}, t)$ derived from the radar data set shown in Figure 1.

where $F_{B N G}^{(3)}(\mathbf{k}, \omega)$ is the spectral energy of the background noise (see Fig. 2). Figure 3 shows a scatter plot of $\sqrt{S N R}$ derived from X-band marine radar data and $H_{s}$ measured by a buoy.

From the estimation of the wave spectrum, and taking into account the different spectral representation of sea states described in the previous Section 2.1, the different sea state parameters can be estimated. Hence, Figure 4 shows a wave number directional spectrum $F^{(2)}(\mathbf{k})$ of a bimodal sea state, where two wave systems (e.g. wind sea and swell) can be identified. This ability of remote sensing imaging techniques to detect multimodal sea states is an advantage compared with the limitations of standard buoys to determine the directionality of wave fields. The following Figure 5 shows the comparison of the frequency spectrum $S(f)$ and the mean direction $\bar{\theta}(f)$ from a marine radar and a directional pitch-roll buoy.

\section{New developments}

The previous sections show the estimation of different wave field properties derived from the wave spectrum. Recent investigations [6] on the analysis of wave fields from X-band marine radars permit us to derive sea surface elevation maps 


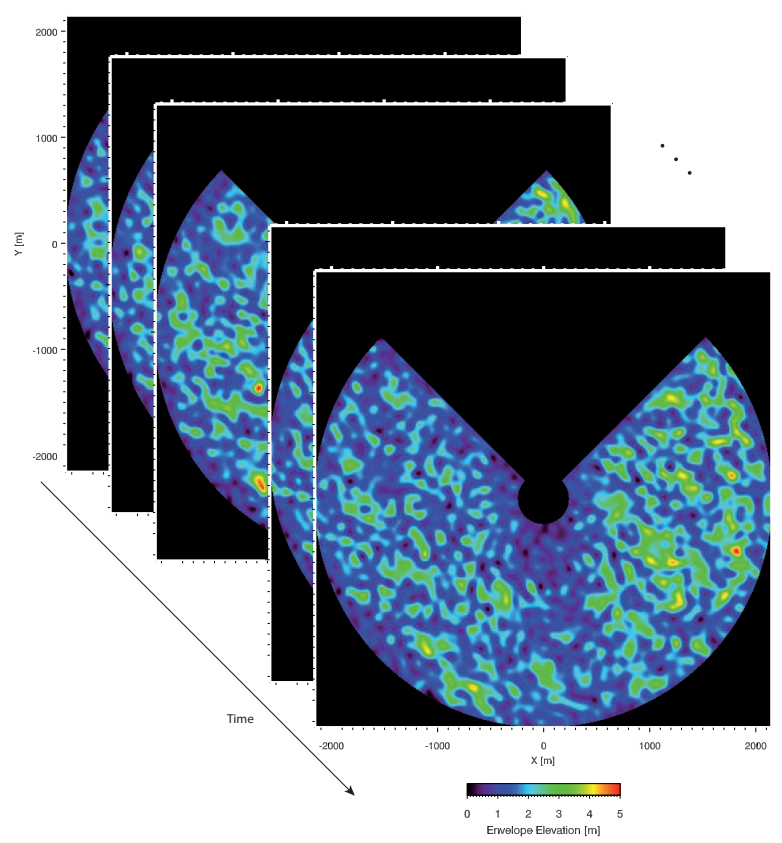

Figure 7: Spatio-temporal evolution of the wave envelope derived from the sea surface estimation shown in Figure 6.

$\eta(\mathbf{r}, t)$. Figure 6 shows an estimation of the wave elevation of the sea surface $\eta(\mathbf{r}, t)$ served from the temporal sequence of radar images shown in Figure 1. From these kinds of data sets individual wave properties in the spatio-temporal domain can be inferred, such as the spatial and temporal evolution of wave groups [19]. This analysis is carried out by estimating the wave envelope in space and time, which is responsible of the energy propagation of wave field. Figure 7 illustrates the estimation of the envelope corresponding to the wave field shown in Figure 6.

\section{Conclusions}

Marine radars are a reliable remote sensing technique to measure and monitor directional sea states and surface currents. These sensors provide a sea clutter image time series of the spatial and temporal variability of surface wave fields. Those images are analyzed and the important sea state parameters can be derived.

One important advantage of marine radars is in the measurement of multidirectional sea states composed of several single swell and wind sea contributions to the total wave field. In these cases, the radar imaging mechanism provides a more detailed directional description than point measurements. So, the information provided by this system can complement, or in some cases substitute, the data obtained from the analysis of conventional in situ sensors. 


\section{Acknowledgements}

The author wants to thank the crew of the vessel Golfo de Vizcaya of the Spanish Maritime Rescue and Safety Institution SASEMAR, where most of the measurements shown in this work were taken from the EXBAYA 95 campaign. In addition, special thanks go to the crew of the German oceanographic vessel Gauss (Bundesamt für Seeschiffahrt und Hydrographie), where some measurements were taken by the author in the ERS-1/2 Tandem experiment and to Dr. F. Ziemer for his support and interest in the two oceanographic experiments mentioned above, and STATOIL, as well as OceanWaveS GmbH for the provision of the marine radar (WaMoS-II) data onboard the FPSO Norne.

\section{References}

[1] Nieto-Borge, J.C. \& Guedes-Soares, C., Analysis of directional wave fields using X-band navigation radar. Coastal Engineering, 40(4), pp. 375-391, 2000.

[2] Nieto-Borge, J.C., Sanz-González, R., Hessner, K., Reichert, K. \& GuedesSoares, C., Estimation of sea state directional spectra by using marine radar imaging of sea surface. Proceedings of ETCE/OMAE2000 Joint Conference Energy for the New Millennium, 1, pp. 1-6, 2000.

[3] Reichert, K., Dannenberg, J., Tränkmann, I. \& Lund, B., X-band radar as a tool to determine spectral and single wave properties. WAVES 2005 Proc ASCE Madrid, 2005.

[4] Izquierdo, P., Guedes-Soares, C. \& Fontes, J., A monitoring of waves with X-band radar in the port of sines. EUROGOSS 2003, 1, pp. 155-160, 2003.

[5] Izquierdo, P. \& Guedes-Soares, C., Analysis of sea waves and wind from X-band radar. Ocean Engineering, 32, pp. 1402-1419, 2005.

[6] Nieto-Borge, J.C., Rodríguez, G., Hessner, K. \& Izquierdo, P., Inversion of marine radar images for surface wave analysis. Jour of Atmos and Ocean Technology, 21(8), pp. 1291-1300, 2004.

[7] Dankert, H. \& Rosenthal, W., Ocean surface determination from Xband radar-image sequences. J Geophysical Res Oceans, 109(C04016), doi:10.1029/2003JC002130, 2004.

[8] Ziemer, F., Brockmann, C., Vaughan, R., Seemann, J. \& Senet, C., Radar survey of near shore bathymetry within the oroma project. EARSeL eProceedings, 3(2), pp. 282-288, 2004.

[9] Nieto-Borge, J.C., Hessner, K., Jarabo-Amores, P. \& de la Mata-Moya, D., Signal-to-noise ratio analysis to estimate ocean wave heights from X-band marine radar image time series. IET Radar, Sonar \& Navigation, 2(1), pp. 35-41, 2008.

[10] Nieto-Borge, J.C., Análisis de campos de oleaje mediante radar de navegación en banda $\mathrm{x}$ (in Spanish). PhD thesis, University of Alcalá, 343 pp., 1997.

[11] Seemann, J., Interpretation der struktur des wellenzahl-frequenzspektrums 
von radar-bildsequenzen (in German). Ph D thesis Universität Hamburg, Hamburg, 1997.

[12] Stevens, C., Poulter, E., Smith, M. \& McCregor, J., Nonlinear features in wave-resolving microwave radar observations of ocean waves. IEEE J Ocean Eng, 24(4), pp. 470-480, 1999.

[13] Young, I.R., Rosenthal, W. \& Ziemer, F., A three dimensional analysis of marine radar images for the determination of ocean waves directionality and surface currents. J Geophys Res, 90, pp. 1049-1059, 1985.

[14] Senet, C., Untersuchungen zur bestimmung der oberflächennahen strömungsgeschwindigkeit mit einen nautischen radar (in German). GKSS 97/E/3, GKSS-Forschungszentrum Geesthacht GMBH, Geesthacht, 1997.

[15] Plant, W., The modulation transfer function. concept and applications. In Radar Scattering from Modulated Wind Waves, Komen and Oost eds, Kluwer Academic Pubs, pp. 155-172, 1989.

[16] Ziemer, F. \& Günther, H., A system to monitor ocean wave fields. Proc of the Second Int Conf on Air-Sea Interaction and Meteorology and Oceanography of the Coastal Zone, Lisbon, Portugal, 1994.

[17] Alpers, W. \& Hasselmann, K., Spectral signal to clutter and thermal noise properties of ocean wave imaging synthetic aperture radars. International Journal of Remote Sensing, 3(4), pp. 423-446, 1982.

[18] Nieto-Borge, J.C., Reichert, K. \& Dittmer, J., Use of nautical radar as a wave monitoring instrument. Coastal Engineering, 37(3-4), pp. 331-342, 1999.

[19] Nieto-Borge, J.C., Reichert, K. \& Hessner, K., Detection of spatio-temporal wave grouping properties by using temporal sequences of $\mathrm{X}$-band radar images of the sea surface. Ocean Modelling, 61, pp. 21-37, 2013. 